\title{
Análisis comparativo de veinte programas de maestrías en educación en Colombia
}

\section{Comparative Analysis of Twenty Master's Education Programs in Colombia}

\author{
Laura Baquero Beltrán, Valentina Hernández Varela, Natalia Torres González, \\ Karen Rangel Vásquez, Nicolás Romero Arévalo, José Castillo Rojas y
}

Oscar Alfonso Huertas

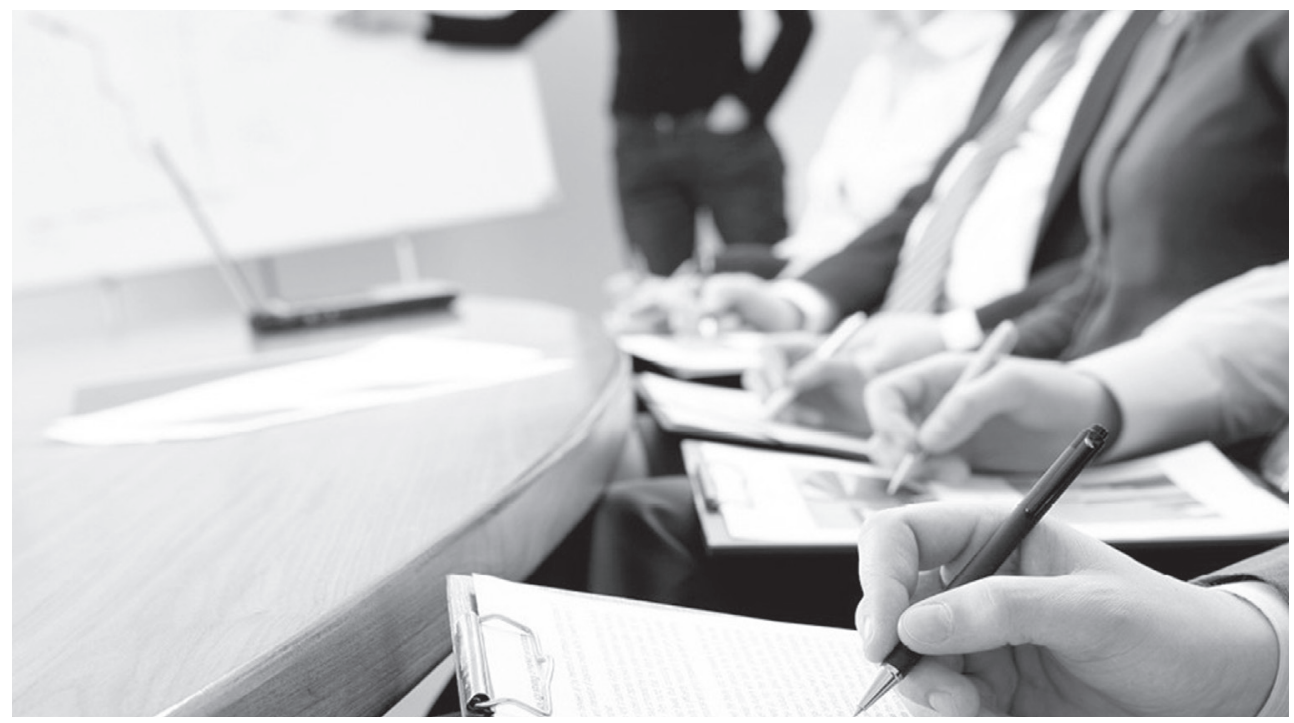

\section{Resumen}

La educación es uno de los pilares principales para alcanzar los objetivos sociales, como la reducción de la pobreza y la mejora de la calidad de vida. En este sentido, los docentes se convierten en uno de los engranajes más importantes del sistema educativo y por tanto es preciso que evalúen diversas variables cualitativas y cuantitativas que les permitan elegir un programa de maestría que cumpla con sus expectativas.

Citar este artículo como: Baquero, L., Hernández, V., Torres, N., Rangel, K., Romero, N., Castillo, J. y Huertas, O. (2016). Análisis comparativo de veinte programas de maestrías en educación en Colombia. Revista Papeles, 8(15), pp. 63-80.

Fecha de recibido: marzo 10 de 2016.

Fecha de aceptación: abril 20 de 2016.

* Estudiantes del curso de Metodología de la investigación I de la Universidad Nacional de Colombia, sede Bogotá, durante el periodo 2016-I. Correos electrónicos: Laura Baquero Beltrán (kvrangelv@unal.edu.co), Valentina Hernández Varela (vhernandezv@unal.edu.co ), Natalia Torres González (nctorresg@unal.edu.co), Karen Rangel Vásquez (kvrangelv@unal.edu.co), Nicolás Romero Arévalo (neromeroa@unal.edu.co), José Castillo Rojas (joscastilloro@unal.edu.co y Oscar Alfonso Huertas (ofalfonsoh@unal.edu.co). 
Este trabajo de investigación pretende ser una guía que permita comparar los aspectos logísticos (como ubicación, modalidad y precio) y aspectos académicos (como enfoques de investigación y profundización, planes de estudios y requisitos de grado) entre veinte universidades colombianas, tanto públicas como privadas, que ofrecen maestrías en educación.

Palabras clave: Colombia, maestrías en educación, docente, público, privado, aspectos logísticos, aspectos académicos.

\begin{abstract}
Education is one of the main pillars to achieve social goals, such as reducing poverty and improving quality of life. In this sense, teachers become one of the most important gears in the educational system and therefore it is necessary to evaluate various qualitative and quantitative variables that allow them to choose a master program that meets their expectations. This research is intended as a guide for comparing the logistical aspects (such as location, modality and price), and academic aspects (such as research approaches and concentration areas of study, curriculum and degree requirements) among twenty Colombian universities, both public and private, that offer master degrees in education.
\end{abstract}

Key words: Colombia, masters of education, teacher, public, private, logistics aspects, academic aspects.

\title{
Introducción
}

La Organización para la Cooperación y el Desarrollo Económico (OCDE, 2013) afirma que la educación no solo permite a los individuos desempeñarse mejor en el mercado de trabajo, sino también contribuye a mejorar su estado de salud, promover el ejercicio de la ciudadanía y la contención de la violencia. Por tanto, es preciso aumentar no solo su cobertura sino también su calidad.

Una de las formas de llevar a cabo la mejora de la calidad de la educación es a través de la oferta de programas de posgrado a los docentes, puesto son éstos quienes requieren de saberes disciplinarios y pedagógicos, para brindar una formación a los educandos de calidad y para proponer nuevas investigaciones que conlleven a resultados positivos para el país. Es por ello que la presente investigación, desarrollada en la asignatura de metodología de la investigación, pretende realizar un análisis comparativo de aspectos logísticos y académicos de maestrías en educación en Colombia.

En tal sentido, se tomó una muestra de veinte programas ofertados en universidades del país, tanto públicas como privadas. Para efectos de esta investigación, se clasificó la información en dos grandes grupos. El primero, relacionado con temas logísticos que corresponden a la ubicación, modalidad y valor de la matrícula de las universidades de la muestra; y el segundo, respecto a temas académicos que corresponden a las variables que inciden en los planes de estudios, enfoques de investigación, líneas de investigación y requisitos para grado.

Así, se pretende ofrecer una guía para los docentes que deseen realizar una maestría en educación en Colombia. La muestra seleccionada corresponde al $10 \%$ del total de las maestrías ofertadas en el país. En total, a la fecha de esta investigación, existen 199 programas vigentes. 


\section{Objetivos}

\section{Objetivo general}

- Realizar un análisis comparativo de variables cualitativas y cuantitativas, que fueron clasificados en aspectos logísticos y académicos de veinte programas de maestrías en educación ofertados en Colombia por universidades, tanto públicas como privadas.

\section{Objetivos específicos}

- Realizar una comparación de los costos de las maestrías en educación en universidades públicas y privadas, haciendo a su vez un análisis de la proporción del PIB per cápita que se debe invertir para acceder a estos programas.

- Identificar los principales ejes en los que se está concentrando la investigación y profundización de los programas de maestría en educación en Colombia.

- Comparar el contenido académico de planes de estudio de los programas de maestría escogidos para esta investigación.

\section{Hipótesis iniciales}

Al iniciar esta investigación se tiene como primera hipótesis que la oferta de maestrías en educación es muy baja respecto a otros estudios de posgrado, debido a que recientemente Colombia se ha ubicado en los últimos puestos de las pruebas PISA que realiza la OCDE. Lo anterior se validará calculando la proporción de las maestrías en educación frente al total de las maestrías ofertadas en el país.

Una segunda hipótesis obedece a que la mayoría de programas de maestría e investigación en educación se ofrecen en Bogotá; dado que, según informe realizado por la OCDE en 2015, aproximadamente el $76 \%$ de la población del país vive en zonas urbanas, siendo Bogotá la ciudad con mayor población.
Por otro lado, se pretende investigar si los costos de estudiar una maestría en educación en Colombia son más bajos que en el exterior y, a su vez, si el costo es más bajo en universidades públicas que en privadas, siguiendo con la tendencia de que los costos de los programas de pregrado son más elevados en universidades privadas. Para esta última premisa se realizó un análisis de los precios de los programas haciendo un promedio de los costos de las universidades públicas y privadas y, del mismo modo, realizando un cálculo del porcentaje del PIB per cápita que se debe invertir para ingresar a una maestría en educación en Colombia, Chile y Perú.

\section{Marco teórico}

Por décadas se ha escuchado que uno de los factores determinantes para el desarrollo de una nación se encuentra en la educación que se brinde al interior de la misma. En este sentido, para efectos de esta investigación, se puede tener como referencia la siguiente definición de educación: "proceso de formación permanente, personal, cultural y social que se fundamenta en una concepción integral de la persona humana, de su dignidad, de sus derechos y de sus deberes" (Ministerio de Educación Nacional, 2016).
Así mismo, en la Constitución Política de Colombia, la educación se establece como un derecho de cada persona y un servicio público que tiene una función social; por ello, se considera como un pilar de desarrollo y un tema de impacto (Secretaría General de la Alcaldía Mayor de Bogotá D.C, 1991).

En cuanto a la definición de maestría en educación, se entiende como el programa que:

Promueve el perfeccionamiento profesional de los docentes y directivos, como 
un proyecto estratégico que direcciona el mejoramiento de la calidad de la educación y tiene en cuenta la satisfacción de la necesidad de formar en competencias para el ejercicio pedagógico e investigativo y la apropiación de tecnologías de la información y la comunicación que promuevan el desarrollo integral en los diferentes campos del actuar humano (Universidad Cooperativa de Colombia, 2016).

\section{Metodología}

La investigación que se plasma en este documento utilizó en gran medida el enfoque de la investigación cualitativa, puesto que implicó la utilización y recolección de una variedad de información, la cual trata de describir algunos aspectos relevantes de las maestrías de educación ofertadas por veinte instituciones de educación superior en Colombia. Así mismo, se cuantificó y se comparó el precio de las maestrías descritas y se calculó el porcentaje del PIB per cápita que se debe invertir

\section{Aspectos logísticos}

\subsection{Generalidades de las maestrías en Colombia}

Sin duda alguna, una de las fuerzas que puede llegar a promover el cambio de un país es la educación que recibe su población. En este sentido, y tal como lo menciona Misas en su libro La educación superior en Colombia, "el lugar estratégico que ocupa actualmente la educación superior, exige a las instituciones y a los programas curriculares y de investigación rendir cuentas ante la sociedad del servicio que prestan". Así pues, en las políticas implantadas por los gobiernos debería siempre estar planteada la pregunta ¿qué tipo de educación superior requiere el país? (Misas, 1994, p.16).

Dado que el objeto de la presente investigación es dar una mirada a las maestrías en educación en Colombia, es conveniente advertir que en el país actualmente existen aproximadamente 2.323 programas de maestrías, de los cuales 199 corresponden a maestrías en educación, que representan el 8,6\% del total ofertado. Lo para ingresar a una maestría en educación en Colombia.

El trabajo se dividió en dos grandes aspectos, como lo son los temas logísticos y los temas académicos. Los primeros agrupan la modalidad, el valor de la matrícula y la ubicación de las universidades. Respecto a los temas académicos se realizó una breve descripción del plan de estudios, las líneas de profundización e investigación de cada plan y el trabajo de grado exigido. anterior ubica a la educación como el cuarto programa con más oferta en Colombia (Guiaacademica.com, 2016) ${ }^{9}$.

Adicionalmente, las 199 maestrías de educación en Colombia se ofertan en cuatro diferentes modalidades, como lo son presencial, semipresencial, a distancia y virtual; siendo la presencial la que tiene mayor auge. El porcentaje de participación de cada modalidad se muestra en la gráfica $1^{10}$ :

9 Para mayor información ver anexo 7.

10 La modalidad presencial consiste en la asistencia a clases dictadas en las instalaciones de las universidades; la modalidad semipresencial corresponde a la asistencia a las instalaciones combinada con clases virtuales; la modalidad a distancia corresponde a una forma de enseñanza en la cual no se requiere asistencia al lugar de estudios sino que se realiza a través de ayudas tecnológicas sin comunicación entre el docente y el estudiante; $y$, finalmente, la modalidad virtual es una educación a distancia la cual permite interacciones entre el docente y el estudiante con ayuda de las Tecnologías de la Información. 
Gráfica 1. Modalidades de maestría en educación

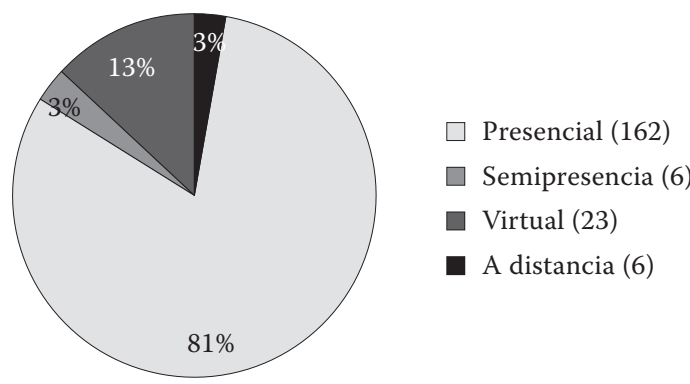

Fuente: elaboración propia

\subsection{Muestra de programas de maestría}

A continuación se relacionan los datos básicos de las veinte universidades consideradas dentro de este estudio, entre las que se realizó una comparación de los programas que ofrecen en cuanto a maestrías en educación.

Con base en la anterior información, se evidencia que de las universidades tomadas en cuenta el $65 \%$ corresponden al sector privado y el restante $35 \%$ se trata de instituciones de

Tabla 1. Información general de instituciones que ofertan maestrías

\begin{tabular}{|c|c|c|c|c|c|c|c|c|}
\hline $\mathbf{N}$ & Nombre institución & Sector & $\begin{array}{l}\text { Fecha de } \\
\text { registro } \\
\text { en SNIES }\end{array}$ & $\begin{array}{c}\text { Cód. } \\
\text { SNIES del } \\
\text { programa }\end{array}$ & Nombre del programa & Modalidad & $\begin{array}{l}\text { Periodos } \\
\text { de } \\
\text { duración }\end{array}$ & $\begin{array}{l}\text { Periodos } \\
\text { de } \\
\text { duración }\end{array}$ \\
\hline 1 & $\begin{array}{l}\text { Corporación Universitaria } \\
\text { Minuto de Dios }\end{array}$ & Privada & $26 / 05 / 2010$ & 90487 & Maestría en educación & Virtual & 5 & Semestral \\
\hline 2 & Pontificia Universidad Javeriana & Privada & $21 / 03 / 1998$ & 1012 & Maestría en educación & Presencial & 4 & Semestral \\
\hline 3 & $\begin{array}{l}\text { Universidad Antonio } \\
\text { Nariño }\end{array}$ & Privada & $14 / 02 / 2014$ & 103085 & Maestría en educación & Presencial & 4 & Semestral \\
\hline 4 & $\begin{array}{l}\text { Universidad Cooperativa } \\
\text { de Colombia }\end{array}$ & Privada & $31 / 12 / 2009$ & 55102 & Maestría en educación & Presencial & 4 & Semestral \\
\hline 5 & Universidad de Antioquia & Oficial & $23 / 02 / 2001$ & 11406 & Maestría en educación & Presencial & 4 & Semestral \\
\hline 6 & Universidad de Caldas & Oficial & $27 / 01 / 2006$ & 51877 & Maestría en educación & Presencial & 2 & Anual \\
\hline 7 & Universidad de la Sabana & Privada & $12 / 01 / 2006$ & 51841 & Maestría en educación & Presencial & 4 & Sin Definir \\
\hline 8 & Universidad de los Andes & Privada & $18 / 12 / 2000$ & 11188 & Maestría en educación & Presencial & 4 & Semestral \\
\hline 9 & Universidad de Manizales & Privada & 09/08/2000 & 10552 & $\begin{array}{c}\text { Maestría en educación y } \\
\text { docencia }\end{array}$ & Presencial & 4 & Semestral \\
\hline 0 & $\begin{array}{l}\text { Universidad de San } \\
\text { Buenaventura }\end{array}$ & Privada & $15 / 01 / 2014$ & 103022 & $\begin{array}{c}\text { Maestría en ciencias de } \\
\text { la educación }\end{array}$ & $\begin{array}{c}\text { Distancia } \\
\text { (tradicional) }\end{array}$ & 4 & Semestral \\
\hline 11 & Universidad del Norte & Privada & 05/10/1999 & 9286 & Maestría en educación & Presencial & 4 & Semestral \\
\hline 12 & $\begin{array}{l}\text { Universidad Distrital Francisco } \\
\text { José de Caldas }\end{array}$ & Oficial & $20 / 01 / 2012$ & 101494 & Maestría en educación & Presencial & 4 & Semestral \\
\hline 13 & Universidad El Bosque & Privada & $13 / 05 / 2011$ & 91326 & $\begin{array}{l}\text { Maestría en docencia de } \\
\text { la educación superior }\end{array}$ & Presencial & 4 & Semestral \\
\hline 14 & $\begin{array}{l}\text { Universidad Externado } \\
\text { de Colombia }\end{array}$ & Privada & $23 / 05 / 2001$ & 12033 & Maestría en educación & Presencial & 4 & Semestral \\
\hline 15 & $\begin{array}{l}\text { Universidad ICESI (Instituto } \\
\text { Colombiano de Estudios Supe- } \\
\text { riores de Incolda) }\end{array}$ & Privada & $14 / 05 / 2012$ & 101666 & Maestría en educación & Presencial & 4 & Semestral \\
\hline 16 & $\begin{array}{l}\text { Universidad Nacional de } \\
\text { Colombia }\end{array}$ & Oficial & $17 / 07 / 2007$ & 52978 & Maestría en educación & Presencial & 4 & Semestral \\
\hline 17 & $\begin{array}{l}\text { Universidad Pedagógica } \\
\text { Nacional }\end{array}$ & Oficial & $21 / 05 / 2002$ & 15903 & Maestría en educación & Presencial & 4 & Semestral \\
\hline 18 & $\begin{array}{l}\text { Universidad Pedagógica y } \\
\text { Tecnológica de Colombia }\end{array}$ & Oficial & $16 / 01 / 2008$ & 53395 & Maestría en educación & Presencial & 4 & Semestral \\
\hline 19 & $\begin{array}{l}\text { Universidad Pontificia } \\
\text { Bolivariana }\end{array}$ & Privada & 08/04/2009 & 54536 & Maestría en educación & Presencial & 2 & Anual \\
\hline 20 & $\begin{array}{l}\text { Universidad Tecnológica de } \\
\text { Pereira }\end{array}$ & Oficial & $14 / 08 / 2007$ & 53027 & Maestría en educación & Presencial & 4 & Semestral \\
\hline
\end{tabular}

Fuente: elaboración propia 
educación superior (IES) pertenecientes al sector público.

Por otro lado, de la muestra escogida se evidencia que un $90 \%$ de las universidades ofrecen su programa en modalidad presencial y que las modalidades virtual y a distancia representan un $5 \%$ cada una.

\subsection{Ubicación}

A continuación se relacionan las ubicaciones de las sedes en las que se ofertan los programas de maestría seleccionados:

Tabla 2. Ubicación de las sedes de maestrías

\begin{tabular}{|c|c|c|c|c|}
\hline $\mathrm{N}$ & Universidad & Departamento & Ciudad & Área \\
\hline \multirow[b]{2}{*}{1} & \multirow{2}{*}{$\begin{array}{l}\text { Corporación Universitaria Minuto de } \\
\text { Dios }\end{array}$} & \multirow[b]{2}{*}{ Cundinamarca } & \multirow[b]{2}{*}{ Bogotá D.C. } & Urbana (DANE, s.a.). \\
\hline & & & & $\begin{array}{l}\text { Metropolitana: reconocida pero no configurada (Cámara de } \\
\text { Representantes, 2012). }\end{array}$ \\
\hline \multirow{2}{*}{2} & \multirow{2}{*}{ Pontificia Universidad Javeriana } & \multirow{2}{*}{ Cundinamarca } & \multirow{2}{*}{ Bogotá D.C. } & Urbana \\
\hline & & & & Metropolitana: reconocida pero no configurada \\
\hline \multirow{2}{*}{3} & \multirow{2}{*}{ Universidad Antonio Nariño } & \multirow{2}{*}{ Cundinamarca } & \multirow{2}{*}{ Bogotá D.C. } & Urbana \\
\hline & & & & Metropolitana: reconocida pero no configurada \\
\hline \multirow{2}{*}{4} & \multirow{2}{*}{ Universidad Cooperativa de Colombia } & \multirow{2}{*}{ Cundinamarca } & \multirow{2}{*}{ Bogotá D.C. } & Urbana \\
\hline & & & & Metropolitana: reconocida pero no configurada \\
\hline \multirow{2}{*}{5} & \multirow{2}{*}{ Universidad de Antioquia } & \multirow{2}{*}{ Antioquia } & \multirow{2}{*}{ Medellín } & Urbana \\
\hline & & & & Metropolitana: reconocida por el acto legislativo 01 de $1968 .{ }^{3}$ \\
\hline \multirow{2}{*}{6} & \multirow{2}{*}{ Universidad de Caldas } & \multirow{2}{*}{ Caldas } & \multirow{2}{*}{ Manizales } & Urbana \\
\hline & & & & Metropolitana: Reconocida pero no configurada \\
\hline \multirow[b]{2}{*}{7} & \multirow{2}{*}{ Universidad de la Sabana } & & & Urbana \\
\hline & & Cundinamarca & Chía & Metropolitana: reconocida pero no configurada \\
\hline & & & & Urbana \\
\hline 8 & Universidad de los Andes & Cundinamarca & Bogotá D.C. & Metropolitana: reconocida pero no configurada \\
\hline & & & & Urbana \\
\hline 9 & Universidad de Manizales & Caldas & Manizales & Metropolitana: Reconocida pero no configurada \\
\hline & & & & Urbana \\
\hline 10 & Universidad de San Buenaventura & Cundinamarca & Bogotá D.C. & Metropolitana: reconocida pero no configurada \\
\hline & & & & Urbana \\
\hline 11 & Universidad del Norte & Atlántico & Barranquilla & Metropolitana: reconocida por el acto legislativo 01 de 1968 \\
\hline & Universidad Distrital Francisco José de & & & Urbana \\
\hline 12 & Caldas & Cundınamarca & Bogotá D.C. & Metropolitana: reconocida pero no configurada. \\
\hline 13 & Universidad Fl Bosaue & Cundinamarca & Boogotá DC & Urbana \\
\hline 13 & Universidad el Bosque & Cundinamarca & Bogota D.C. & Metropolitana: reconocida pero no configurada \\
\hline & & & & Urbana \\
\hline 14 & Universidad Externado de Colombia & Cundinamarca & Bogotá D.C. & Metropolitana: reconocida pero no configurada \\
\hline & Universidad ICESI (Instituto Colom- & Valle del & Santiago de & Urbana \\
\hline 15 & biano de Estudios Superiores de Incolda) & Cauca & Cali & Metropolitana: Reconocida pero no configurada \\
\hline & & & & Urbana \\
\hline 16 & Universidad Nacional de Colombia & Cundinamarca & Bogota D.C. & Metropolitana: reconocida pero no configurada \\
\hline & & & & Urbana \\
\hline 17 & Universidad Pedagógica Nacional & Cundinamarca & Bogotá D.C. & Metropolitana: reconocida pero no configurada \\
\hline & Universidad Pedagógica y Tecnológica & & & Urbana \\
\hline 18 & de Colombia & Boyacá & Tunja & Metropolitana: reconocida pero no configurada \\
\hline & & & & Urbana \\
\hline 19 & Universidad Pontificia Bolivariana & Antioquia & Medellin & Metropolitana: Reconocida pero no configurada \\
\hline 20 & Unixersidad Tecnológica de Pereir & Risaralda & Pereirn & Urbana \\
\hline 20 & Universidad lecnologica de Pereira & Risaralda & Pereira & Metropolitana: Reconocida por el acto legislativo 01 de 1968 \\
\hline
\end{tabular}

Fuente: elaboración propia 
De acuerdo a la información presentada, a continuación se relaciona la concentración de cada uno de los programas por departamento, que refleja que el $50 \%$ de maestrías en educación se concentra en la capital del país y el 50\% restante en las demás ciudades del país.

Gráfica 2. Concentración de maestrías en educación por departamentos

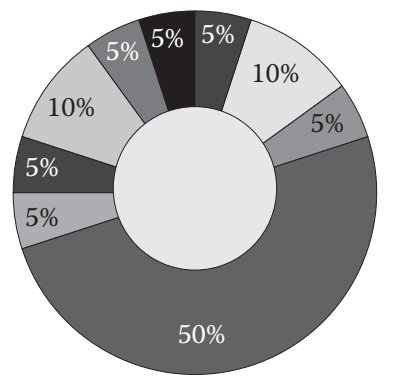

$$
\begin{aligned}
& \square \text { Antioquia } \\
& \square \text { Atlántico } \\
& \square \text { Bogotá, D.C. } \\
& \square \text { Bolívar } \\
& \square \text { Boyacá } \\
& \square \text { Caldas } \\
& \square \text { Cundinamarca } \\
& \square \text { Risaralda } \\
& \square \text { Valle del Cauca }
\end{aligned}
$$

Fuente: elaboración propia

\subsection{Análisis de precios}

Como primera instancia se tomaron los datos pertinentes respecto al precio de todos los programas, con el valor vigente para el semestre 2016-II. Se debe tener en cuenta que son valores aproximados, pues no todas las universidades establecen precios fijos, sino que, por ejemplo, algunas informan el costo por cada crédito que se inscriba, y muchas otras ofertan descuentos o métodos de financiación que afectan el valor. Los resultados se pueden apreciar en la gráfica 3 (ver anexo 1).

Estos datos arrojaron un promedio de costo semestral, en programas de maestrías en educación, de $\$ 5^{\prime} 191.408$, y un costo total promedio de $\$ 20$ '940.984. No obstante, al momento de realizar un análisis más profundo y comparar los valores, teniendo como premisa el carácter público o privado de la institución, los resultados sufren una gran variación.

Las instituciones públicas tienen un costo promedio semestral de $\$ 4 ' 131.369$ y un costo promedio total de $\$ 16^{\prime} 525.474$, mientras que en aquellas de carácter privado un semestre promedio cuesta $\$ 5^{\prime} 762.202$, y la totalidad del programa es de $\$ 23 ’ 048.806$. En conclusión, los programas de carácter público son más económicos que aquellos de carácter privado

$\$ 10,000,000$

Gráfica 3. Análisis de precios

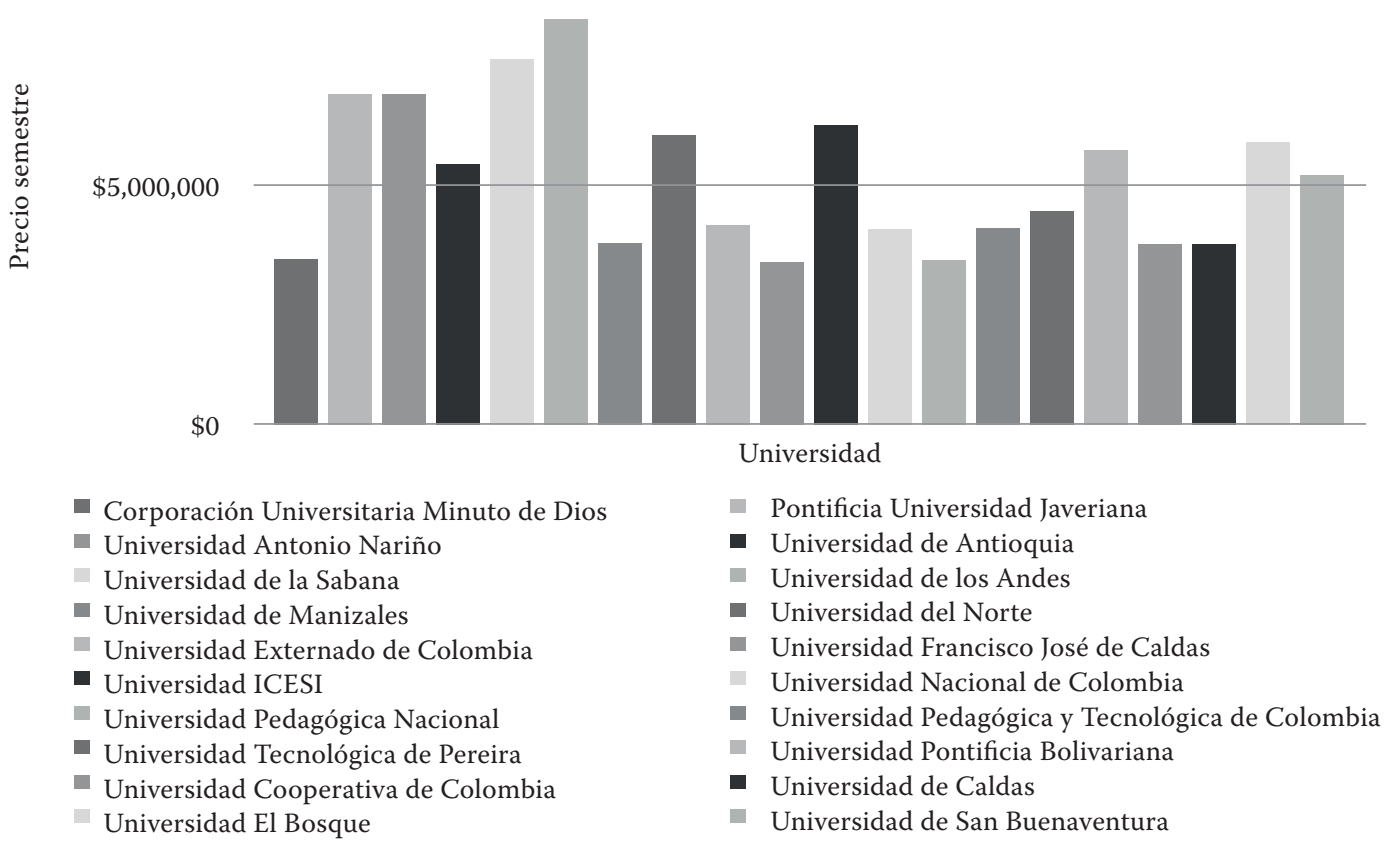

Fuente: elaboración propia 
en un $27 \%$, continuando con el patrón de los costos en pregrado en menor proporción. Por otro lado, si se evalúan las diferentes posibilidades de ayuda financiera (como descuentos) brindadas por las instituciones de educación superior, la mayoría están encaminadas para ser ofrecidas a estudiantes que ya han cursado su pregrado en dicha institución; por ende, las instituciones trabajan con suposiciones acerca de cuál es la capacidad de pago de los grupos a los que principalmente son ofertados los programas. Es por esta razón que los programas públicos mantienen sus costos bajos, e incluso ofrecen otro tipo de descuentos relacionados, por ejemplo, con la presentación del certificado electoral o la pertenencia a grupos vulnerables de la población (ver anexo 2).

Como último criterio de análisis de precios se evaluó cuál es el impacto en el ingreso de un colombiano promedio por el pago de una maestría dependiendo del carácter público o privado de la institución. Para evidenciar este costo se tomaron cifras del Banco Mundial y se obtuvo el PIB per cápita en pesos constantes. Como esta información es anual, el porcentaje corresponde a lo que una persona destinaría por año, es decir, el valor de dos semestres del programa. Adicionalmente, como criterio de comparación se realizó el mismo proceso con programas de maestrías en educación, públicas y privadas, en Chile y Perú. (Ver anexos 3, 4, 5 y 6).

\section{Ley 30 de 1992, solo indica que el} aumento de las matrículas se debe realizar por el índice de precios del consumidor, sin embargo, la ley aclara que las instituciones privadas están en la plena libertad de hacer aumentos por encima de este índice si lo justifican ante el Ministerio de Educación
Gráfica 4. Porcentaje de BIB percápita

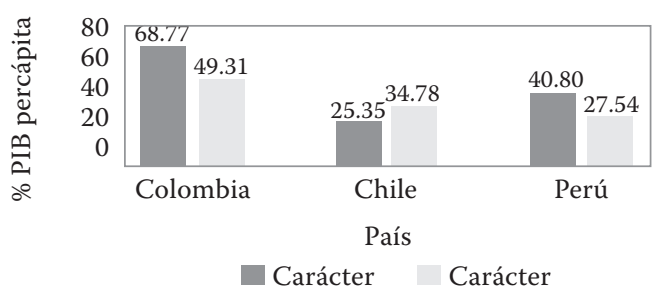

Fuente: elaboración propia

La gráfica permite concluir los altos costos de la educación en Colombia a nivel público y privado: una persona debería destinar en promedio un 58,43\% de su ingreso anual para educación, una cifra que evidencia la poca preocupación por parte del Estado en hacer de la educación un servicio accesible a la población común; ya que los altos precios que cobran la mayoría de las universidades llevan a bajos niveles de inscripción o, en su defecto, a endeudamientos y al sacrificio significativo en el nivel de vida de las personas (acceso a entretenimiento, inversiones futuras, etc.).

De acuerdo a lo anterior, la despreocupación del gobierno en los altos costos de las matrículas se ve reflejada en la autonomía que tienen actualmente las instituciones privadas para establecerlos. En este sentido, la Ley 30 de 1992, solo indica que el aumento de las matrículas se debe realizar por el índice de precios del consumidor, sin embargo, la ley aclara que las instituciones privadas están en la plena libertad de hacer aumentos por encima de este índice si lo justifican ante el Ministerio de Educación (Revista Semana, 2016).

Finalmente, el estado actual del acceso a la educación superior agudiza la brecha entre las personas que tienen acceso a los programas educativos y aquellos que no, aportando en las cifras de desigualdad del país; puesto que "tener una educación universitaria sin duda constituye una ventaja para cualquier ciudadano, dada la importancia del conocimiento, las habilidades técnicas y el acceso a una red privilegiada para efectos laborales y sociales" (Gómez, 2016). 


\section{Aspectos académicos}

\subsection{Enfoques de investigación y profundización}

Con base en la información contenida en la página web de cada universidad, a continuación se presenta una síntesis de los enfoques de investigación y profundización de cada programa de maestría.

\section{Corporación Universitaria Minuto de Dios}

Esta maestría en educación se brinda a distancia, de forma virtual y en cinco períodos, siendo la única con estas características dentro de la comparación que se realiza en el presente trabajo. Así, igualmente, y de forma única, al acceder a este programa, se accede a un doble grado académico, en el que el egresado saldrá titulado como Magíster en Educación por la UNIMINUTO, en Colombia, y como Maestro en Educación por el Tecnológico de Monterrey, en México. Sus líneas de investigación son praxeología pedagógica, impacto social de los modelos educativos innovadores, uso de las tecnologías en la educación, modelos innovadores de gestión educativa, didácticas específicas, pedagogía social con opción por el desarrollo integral, modelos y procesos innovadores en la enseñanza-aprendizaje.

\section{Pontificia Universidad Javeriana}

Los requisitos para acceder a esta maestría son: tener habilidades de trabajo en equipo, comunicativas y escriturales para la elaboración de artículos; comprensión lectora en un segundo idioma, y acreditar una experiencia mínima de un año en el campo educativo (evaluados mediante recomendaciones académicas, entrevistas y valoración de un escrito académico presentado). Adicionalmente, se desarrollan talleres de formación en investigación educativa, como herramientas para el conocimiento de los fundamentos epistemológicos de la investigación, sus usos y aplicaciones.

\section{Universidad Antonio Nariño}

Con un enfoque totalmente investigativo, se orienta a la educación ambiental, el uso de las tecnologías de la información y la comunicación en educación, la educación y la sociedad, y la administración educativa. A partir de estas líneas de profundización, un egresado de la UAN podrá desempeñarse como líder, coordinador y docente investigador.

\section{Universidad Cooperativa de Colombia}

Esta maestría en educación brinda la posibilidad de desarrollar este programa académico de forma virtual. El programa se destaca por brindar en el proceso de formación herramientas que comprenden la cibernética social, a través de la metodología MICEA; el constructivismo, el pensamiento complejo y la neurociencia. Existen cuatro líneas temáticas para orientar los trabajos de grado: estrategias de enseñanza y aprendizaje, gestión de la calidad educativa, dificultades del aprendizaje y educación mediada por las TIC.

\section{Universidad de Antioquia}

La particularidad de esta maestría radica en que, como requisito de inscripción, es obligatorio la presentación de un proyecto inicial, no superior a diez hojas y que tendrá que ser aprobado por los pares académicos para poder iniciar el proceso de selección al interior de la maestría. El programa se concentra en temas tales como educación matemática, estudios curriculares, educación y tecnologías de la información y la comunicación, formación de maestros, pedagogía y diversidad cultural, estudios educativos sobre cognición y creatividad, educación en ciencias sociales y formación ciudadana, enseñanza de la lengua y de la literatura, pedagogía social, didáctica de la geografía, democracia y convivencia escolar, y didáctica de la educación superior. 


\section{Universidad de Caldas}

Para graduarse del programa de maestría en educación en esta universidad, los estudiantes deben elegir, desde el inicio como guía de su posgrado, entre dos modalidades: investigación, que requiere de un trabajo de investigación sustentado y aprobado para graduarse; o profundización, que comprende énfasis en ciencias sociales, énfasis en lengua castellana y énfasis en filosofía, y para el cual es necesario realizar una tesis de grado de intervención educativa en la propia institución laboral; pues es requisito de esta modalidad que la tesis impacte en el PEI de la institución en la que desarrolla su actividad profesional el aspirante a magíster en educación.

\section{Universidad de la Sabana}

Su enfoque es la investigación socio-educativa, donde se trabaja en la solución de problemáticas educativas que repercuten en el desarrollo de la sociedad. Se trabaja paralelamente el campo investigativo y de conceptualización, siendo el primero el de mayor peso e importancia en la formación del egresado, pues guía el trabajo de grado; sin embargo, la conceptualización también es relevante, pues orienta al estudiante en cuanto a política educativa, pedagogía social e instituciones educativas.

\section{Universidad de los Andes}

Esta maestría plantea unas áreas de concentración tales como currículo y pedagogía; gestión educativa y políticas públicas en educación; educación para el bilingüismo; educación en ciencia, tecnología, ingeniería y matemática (CTIM); educación y tecnologías de información y comunicación; educación para la ciudadanía; y educación a la primera infancia.

\section{Universidad de Manizales}

La Universidad de Manizales presenta tres programas de maestría relativos a la educación, como son maestría en educación para docencia, maestría en educación y desarrollo humano y maestría en educación desde la diversidad. Esta última se brinda a distancia.

La universidad se caracteriza por adoptar una postura crítica, que se inculca a sus estudiantes. Así mismo, trata de generar motivación hacia la reflexión, buscando impartir una educación no convencional. Por otra parte, trabaja con dos énfasis generales de formación en investigación y profundización. Finalmente, aunque su metodología es presencial, brinda asistencia virtual para apoyar a los participantes en el desarrollo del programa.

\section{Universidad de San Buenaventura}

$\mathrm{Su}$ modalidad es presencial. Se desarrolla mediante procesos de investigación o profundización, según elija el estudiante, con el fin de aportar a la teorización y problematización en torno a la pregunta por la formación, la educación, la sociedad y la cultura. El programa cuenta con el respaldo de dos grupos de investigación: GIDEP "Grupo Interdisciplinario de Estudios Pedagógicos (clasificado por Colciencias en la categoría B) y ESINED "Grupo de estudios interdisciplinarios sobre educación" (clasificado por Colciencias en la categoría B); y desarrolla las siguientes líneas y grupos de investigación: desarrollo humano y contextos educativos, infancia, pedagogía, lenguaje y comunicación, gestión educativa-currículo, didáctica de las ciencias, educación física, cuerpo y motricidad, estudios culturales, y lenguajes contemporáneos.

\section{Universidad del Norte}

Cuenta con una modalidad de investigación y una de profundización, donde sus enfoques de investigación son desarrollados por medio de grupos de trabajo en cinco áreas, a saber: cognición y educación, educación infantil, medios aplicados a la educación, lenguaje y prácticas pedagógicas y pensamiento matemático. 


\section{Universidad Distrital Francisco José de Caldas}

Con modalidades de formación de profundización e investigación, se enfoca en la educación en matemática, ciencias de la naturaleza y tecnología, en comunicación intercultural, etnoeducación y diversidad cultural, en ciencias sociales (ética, política), y educación.

\section{Universidad El Bosque}

Es de modalidad presencial con apoyo de aulas virtuales. Comprende diversas estrategias didácticas, como la cátedra, el taller, el seminario de profundización y el trabajo de investigación con tutoría y dirección. Comprende dos grupos de soporte de investigación: 1) Educación e Investigación UNBOSQUE (categoría C de Colciencias), que a su vez comprende cinco líneas de investigación: pedagogía universitaria, didáctica de la educación superior, concepción, diseño y gestión del currículo; evaluación de la educación superior, y tecnologías de la información aplicadas a la educación. 2) Grupo RUECA (Evaluación de la Calidad de la Educación-Capítulo Colombia, categoría A de Colciencias), que aborda la calidad de la educación como línea de investigación.

\section{Universidad Externado de Colombia}

Presenta dos programas de maestría relativos a la educación: maestría en educación, en la modalidad de investigación; y maestría en evaluación y aseguramiento de la calidad de la educación. Se realiza una formación complementaria en la que se resaltan los seminarios de profundización, donde se trabaja el énfasis de investigación que el estudiante haya seleccionado. Adicionalmente, incluye un componente de teorización, que le da importancia tanto a la instrucción pedagógica como cultural, para contribuir a una formación integral del aspirante a magíster en educación. Sus énfasis de investigación son aprendizaje de la lectoescritura y las matemáticas, gestión educativa y evaluación, desarrollo humano y valores, pedagogía de las ciencias sociales. y didáctica del inglés.

\section{Instituto Colombiano de Estudios Superiores de Incolda}

Este programa cuenta con tres grandes líneas de investigación: prácticas pedagógicas, competencias e innovación escolar, y convivencia y conflicto escolar. Adicionalmente, hace uso de las TIC para los procesos de enseñanza y aprendizaje. Su modalidad es semipresencial con encuentros cada quince días (viernes y sábado), y está respaldada por el Centro de Recursos para el Aprendizaje de la Universidad ICESI, pionero en el uso de didácticas basadas en la filosofía del aprendizaje activo.

\section{Universidad Nacional de Colombia}

La maestría de educación desarrollada presenta dos tipos de perfiles a escoger. En primer lugar, la profundización/profesionalización, donde el egresado se caracteriza por la reflexión crítica y la capacidad de diseñar y desarrollar proyectos de innovación pedagógica y educativa. En segundo lugar, la investigación, en la que el egresado será capaz de abordar los problemas de la educación a partir de la actividad y la experiencia educativa. Sus líneas de investigación son lenguajes y literaturas en educación, comunicación y educación, ciencias sociales y educación, y pedagogía y docencia universitaria.

\section{Universidad Pedagógica Nacional de Colombia}

Este programa tiene un aspecto que lo diferencia, puesto que académicamente se estructura por grupos de investigación con reconocimiento nacional e internacional, con una importante productividad que se refleja en la participación de un buen número de eventos, así como publicaciones de libros y artículos. En este sentido, esta propuesta ha introducido significativas modificaciones en la formación 
avanzada en Colombia, ya que ha venido generando producción de conocimiento educativo en dimensiones culturales, sociales, éticas y políticas desde que el estudiante ingresa a cursar el programa (Universidad Pedagógica Nacional, 2016).

Los énfasis de investigación son: historia pedagogía y cultura política, evaluación y gestión educativa, educación comunitaria, cultura política e interculturalidad, educación superior, conocimiento y comunicación, política pública educativa, y desarrollo regional.

\section{Universidad Pedagógica y Tecnológica de Colombia}

Este programa se brinda tanto en modalidad de investigación como de profundización. Para el primer caso, se da mayor importancia a las corrientes epistemológicas que han desarrollado la educación en el país y sus repercusiones de otras áreas: antropología, psicología, sociología, entre otras, en el desarrollo del proceso pedagógico. Para el segundo caso, los seminarios disciplinares en el área pedagógica son los que marcan el desarrollo del proceso formativo, paralelo a la formación investigativa. Sus enfoques giran en torno a la educación matemática, creación y pedagogía, docencia universitaria, pedagogía y currículo, filosofía de la educación y la enseñanza de la filosofía, tecnologías de la información y la comunicación, y emociones y educación.

\section{Universidad Pontificia Bolivariana}

La tesis del estudiante que curse esta maestría debe reflejar la adquisición de competencias científicas propias de un investigador académico, y cuya innovación se deriva de la aplicación de los resultados de los grupos de investigación propios de la Escuela de educación y pedagogía.

La Maestría contempla dos rutas de formación: ambientes de aprendizaje mediados por las TIC y maestro: pensamiento-formación. Estas rutas de formación se sustentan en dos grupos de investigación escalafonados por Colciencias: Educación en Ambientes Virtuales (EAV) y Didáctica de los Saberes (PDS), que se desarrollan mediante macroproyectos.

\section{Universidad Tecnológica de Pereira}

Para obtener el título de Magíster en Educación es necesario desarrollar un proyecto de investigación trabajando el enfoque seleccionado por el estudiante. La universidad brinda los siguientes enfoques: escuela, conflicto y sociedad; didáctica del lenguaje; y didáctica de las ciencias naturales y exactas.

\subsection{Plan de estudios de las maestrías}

Para analizar el plan de estudios de la muestra seleccionada, se agruparon en cinco (5) categorías las materias obligatorias de cada maestría, de la siguiente manera:

- Fundamentación en educación tradicional: en esta categoría se concentran todas las materias de carácter epistemológico, filosófico, psicológico y pedagógico que fundamentan la disciplina educativa $y$ que han sido tradicional en las escuelas y universidades. No se realizó una discriminación de la disciplina de las mismas (es decir, si son matemáticas, ciencias sociales, historia, etc.).

- Política pública y gestión educativa: como su nombre lo indica, hace referencia a una parte más administrativa y relacionada con las políticas de carácter educativo.

- Ciencia, tecnología e innovación: se agruparon las materias relacionadas con nuevas tecnologías de carácter global y la educación, de igual manera las relacionadas con la innovación en nuevos métodos de enseñanza.

- Investigación: hace referencia a seminarios y materias orientadas netamente a la investigación en su conjunto. 
- Formación ética: estas materias hacen referencia a aquellas orientadas a la fundamentación ética del magíster para desarrollar estrategias de este carácter y de resolución de conflictos al interior de las aulas.

A continuación se detalla la distribución de los cursos según las categorías enunciadas anteriormente:

Gráfica 5. Distribución de maestrías en educación por plan de estudios

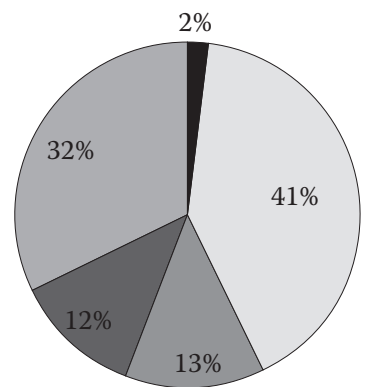

$\square$ Fundamentación educación tradicional

$\square$ Políticas públicas educativas y gerencia

- Ciencia, tecnología e innovación

$\square$ Investigación

Fuente: elaboración propia

Respecto a los datos arrojados se puede observar que:

- Se resalta que la mayoría de materias que se ofrecen en los postgrados son de fundamentación en educación tradicional, es decir, materias que han sido ofertadas usualmente en los programas de maestría (41\%); no obstante, las materias relacionadas con las nuevas tecnologías, que promulguen la innovación y el desarrollo de las técnicas pedagógicas, están en desarrollo (actualmente $12 \%)$.

- Se evidencia la creciente tendencia que han experimentado las maestrías respecto a nuevos enfoques orientados a las condiciones sociales venideras para Colombia, como es el caso del postconflicto.

\subsection{Requisitos de grado}

Respecto a los requisitos de grado exigidos por cada una de las instituciones de educación superior, se encontraron tres modalidades: tesis, trabajos de investigación y proyectos prácticos que buscan solucionar un problema. La siguiente gráfica indica el porcentaje de universidades que exige cada requisito de grado:

Gráfica 6. Requisitos de grado

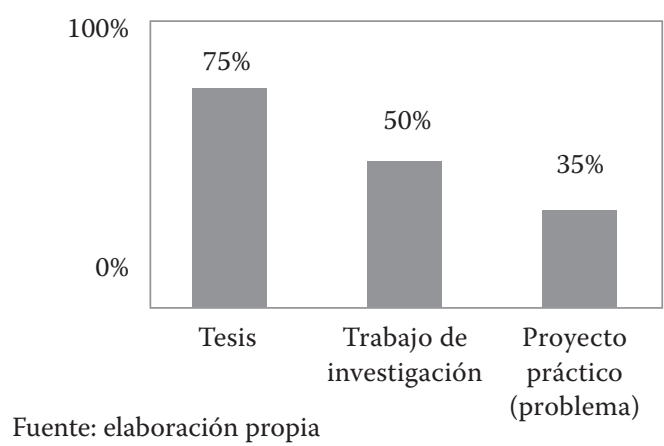

Dentro de las universidades de la muestra se identificó que la Universidad de los Andes y la Universidad El Bosque tienen como requisitos de trabajo de grado las tres opciones descritas anteriormente. La Universidad Tecnológica de Pereira, la Universidad Externado de Colombia, la Universidad de Manizales y la Universidad de Caldas tienen como opción de grado tanto tesis como trabajo de investigación. El resto de universidades solo tiene una opción para realizar el trabajo de grado.

\section{Conclusiones}

Frente a las hipótesis establecidas y los resultados obtenidos en esta investigación, se puede concluir que la educación es el cuarto programa de maestría con más oferta en el país. Así, bajo la muestra realizada, la concentración de la oferta se concentra en la capital en un $50 \%$ y el $50 \%$ restante se distribuye entre las demás ciudades del país.

Adicionalmente, en Colombia se mantiene la tendencia a la modalidad presencial y con 
programas que ofertan en gran medida materias categorizadas como de fundamentación en educación tradicional e investigación, reflejando una gran oportunidad de mejora en el uso de las tecnologías de la información e innovación.

Por otro lado, concluimos que los programas de carácter público son más económicos que aquellos de carácter privado, continuando con el patrón de los costos en pregrado; y que a pesar de lo anterior, los programas de maestría en educación en Colombia son más costosos que en otros países latinoamericanos como Chile y Perú.

Finalmente, fue evidente que los planes de estudios y los enfoques de investigación están concentrados en temáticas tradicionales de la educación. Sin embargo, se espera que se promueva el fortalecimiento de la innovación, ciencia y tecnología.

\section{Referencias}

Cámara de Representantes. (2012). Proyecto de Acto Legislativo 088 de 2012 Cámara. Bogotá. Recuperado de file://C:/Users/ntorres/ Downloads/PROYECTO\%20DE\%20ACTO\%20 LEGISLATIVO\%20088\%20DE\%202012\%20 C\%C3\%81MARA.pdf

Corporación Universitaria Minuto de Dios. (2016). Programas Académicos Uniminuto. Bogotá. Recuperado de http://www.uniminuto. edu/web/programasacademicos/maestrias/-

DANE. (S.a.). Conceptos Básicos. Bogotá. Recuperado de http://www.dane.gov.co/files/ inf_geo/4Ge_ConceptosBasicos.pdf.

DANE. (2016). DANE. Bogotá. Recuperado de http://www.dane.gov.co/index.php/esp/com ponent/content/article/144-noticias / noticias/6523-pib-oferta-iv-trimestre-2015

Gómez, S. C. (2016). El precio de la matrícula no es el problema. Bogotá. Recuperado de http://www.eltiempo.com/economia/finan zas-personales/el-precio-de-la-matricula-no-esel-problema/16506836

Guía académica. (2016). Guíaacademica.com. Bogotá. Recuperado de http://www.guiaacademica.com/educacion/carreras-posgrados/ universidades/

Instituto Nacional de Estadística e Informática. (2016). Series. Bogotá. Recuperado de http:// series.inei.gob.pe:8080/sirtod-series/

Instituto Nacional de Estadísticas Chile. (2016). Instituto Nacional de Estadísticas Chile. Recuperado de http://www.ine.cl/
Ministerio de Educación Nacional. (2009) ¿Qué es la educación superior? Recuperado de: http://www.mineducacion.gov.co/1621/arti cle-196477.html

Ministerio de Educación Nacional (2016) Sistema Nacional de Información de la Educación Superior. Recuperado de http://www.mineducacion.gov.co/sistemasdeinformacion/1735/ w3-propertyname-2672.html

Misas, A. G. (1994). La educación superior en Colombia. Bogotá. Colombia: Universidad Nacional de Colombia.

OCDE. (2013). Education Indicators in Focus. Recuperado de http://www.mecd.gob.es/dctm /inee/indicator-in-focus/edif-2012--n10esp.pdf? documentId=0901e72b816cba2f.

ONU. (2016). United Nations Statics Division. New York, United States of America. Recuperado de http://www.un.org/es/index.html

Pontificia Universidad Católica de Chile. (2016). Pontificia Universidad Católica de Chile. Chile. Recuperado de http://educacion.uc.cl/ 2015-01-08-21-56-40/magister-en-educacion

Pontificia Universidad Católica de Perú. (2016). Pontificia Universidad Católica de Perú. Recuperado de http://www.pucp.edu.pe/

Pontificia Universidad Católica de Valparaíso. (2016). Pontificia Universidad Católica de Valparaíso. Recuperado de http://www.pucv. $\mathrm{cl} /$ pucv/facultad-de-filosofia-y-educacion/ postgrados/magister-en-educacion/magis ter-en-educacion/2015-06-30/123637.html 
Pontificia Universidad Javeriana. (2016). Maestría-educación. Bogotá. Recuperado de http:// www.javeriana.edu.co/maestria-educacion

Revista Semana. (2016). Las matrículas universitarias cada vez más altas. Bogotá. Recuperado de http://www.semana.com/educacion/ articulo/los-altos-precios-de-las-universidadesen-colombia/457514-3

Secretaría General de la Alcaldía Mayor de Bogotá D.C. (1991). Constitución Política de Colombia. Recuperado de http://www.alcaldiabogota.gov.co/sisjur/normas/Norma1

Universidad Antonio Nariño. (2016). Universidad Antonio Nariño. Bogotá. Recu perado de http://www.uan.edu.co/maestria-en-educacion

Universidad Cooperativa de Colombia. (2016). Universidad Cooperativa de Colombia. Bogotá. Recuperado de http://www.ucc.edu.co/bogota/ programas/Paginas/maestria-educacion-bogota. aspx

Universidad de Antioquia. (2016). Portaludea. Medellín. Recuperado de http://portal.udea.edu. co/wps/portal/udea/web/inicio/institucional/ unidades-academicas/facultades/educacion

Universidad de Caldas. (2016). Maestría en educación. Manizales. Recuperado de http://www. ucaldas.edu.co/portal/maestria-en-educacion/

Universidad de Chile. (2016). Posgrados. Chile. Recuperado de http://www.uchile.cl/postgrados /22026/educacion-mencion-en-curriculo-ycomunidad-educativa

Universidad de Concepción. (2016). Facultad de Educación Universidad de Concepción. Chile. Recuperado de http://educacion.udec.cl/

Universidad de la Frontera. (2016). Universidad de la Frontera. Chile. Recuperado de http://huma nidades.ufro.cl/

Universidad de la Sabana. (2016). Posgrados. Bogotá. Recuperado de http://www.unisabana. edu.co/postgrados/maestria-en-educacion/ nuestro-programa/

Universidad de los Andes. (2016). Universidad de los Andes Facultad de Educación. Bogotá. Recuperado de: https://cife.uniandes. edu.co/index.php/programas/maestria/ maestria-en-educacion

Universidad de Manizales. (2016). Maestría en Educación y docencia. Manizales. Recuperado de http://www.umanizales.edu.co/u/carreras/pos grados/mae_educacion_docencia/

Universidad de Santiago de Chile. (2016). Dirección de posgrados. Chile. Recuperado de http://www.postgrado.usach.cl/es/programas -de-estudios/magister-en-educacion

Universidad del Tolima. (2016) Maestría en educación. Ibagué. Recuperado de http://idead. ut.edu.co/index.php/oferta-academica-idead/ proceso-de-inscripcion-pregrado/8-demo/654oferta-academica-semestre-a-de-2016

Universidad del Norte. (2016). Maestría en Educación. Bogotá. Recuperado de http://www. umanizales.edu.co/u/carreras/posgrados/ mae_educacion_docencia/

Universidad Distrital Francisco José de Caldas. (2016). Maestría en educación. Bogotá. Recuperado de http://maestriaeducacion.udistrital.edu. co:8080/

Universidad el bosque. (2016) Maestría en educación. Bogotá. Recuperado de http://www. uelbosque.edu.co/programas_academicos/ maestrias/docencia_educacion_superior

Universidad Externado de Colombia. (2016). Universidad Externado de Colombia. Bogotá. Recuperado de http://www.uexternado.edu.co/ esp/posgrado/

Universidad ICESI. (2016). Universidad ICESI. Bogotá. Recuperado de http://www.icesi.edu.co/ maestrias/educacion/

Universidad Nacional de Colombia. (2016). Instituto de investigación en Educación. Bogotá. Recuperado de http://www.humanas.unal.edu. co/iedu/programas-curriculares/

Universidad Nacional de Trujillo. (2016). Universidad Nacional de Trujillo. Perú. Recuperado de http://pg.unitru.edu.pe/

Universidad Nacional Mayor de San Marcos. (2016). Universidad Nacional Mayor de San Marcos. Perú. Recuperado de http://epg.unm sm.edu.pe/index.php/maestria/humanida des-y-ciencias-sociales/educacion 
Universidad Nacional San Antonio Abad del Cusco. (2016). Universidad Nacional San Antonio Abad del Cusco. Perú. Recuperado de http:// postgrado.unsaac.edu.pe/laescuela/

Universidad Pedagógica Nacional. (2016). Posgrados. Bogotá. Recuperado de http://educa cion.pedagogica.edu.co/vercontenido.php? idp $=407 \&$ idh $=412$

Universidad Pedagógica Tecnológica de Colombia. (2016). Universidad Pedagógica Tecnológica de Colombia. Bogotá. Recuperado de http:// www.uptc.edu.co/facultades/f_educacion/maestria/educacion/inf_general/index.html

Universidad Peruana Cayetano Heredia. (2016). Universidad Peruana Cayetano Heredia. Perú. Recuperado de http://www.upch.edu. pe/epgvac/maestria/136/maestria-en-educacion-con-mencion-en-docencia-e-investigacion-en-educacion-superior
Universidad Pontificia Bolivariana (2016). Universidad Pontificia Bolivariana. Medellín. Recuperado de http://www.upb.edu.co/portal/ page?_pageid=1054,36089540\&_dad=portal

Universidad San Buenaventura. (2016). Maestría en ciencias de la educación. Bogotá. Recuperado de http://www.usbbog.edu.co/facul tad-ciencias-humanas-sociales-16/maestria -en-ciencias-de-la-educacion

Universidad Señor de Sipan. (2016). Universidad Señor de Sipan. Perú. Recuperado de http:// www.uss.edu.pe/uss/Descargas/1044/Archivos/ CIENCIASDELAEDUCACIONCONMENCIONENGESTIONEDUCATIVA.pdf

Universidad Tecnológica de Pereira. (2016). Universidad Tecnológica de Pereira. Pereira. Recuperado de http://www.utp.edu.co/registro /posgrado/203/maestria-en-educacion

\section{Anexos}

Anexo 1. Tabla 3. Precios maestrías en educación

\begin{tabular}{lcllllll}
\hline \multicolumn{1}{c}{ Institución } & Ciudad & Programa & Carácter & Duración & $\begin{array}{c}\text { Precio } \\
\text { Semestral }\end{array}$ & $\begin{array}{c}\text { Precio } \\
\text { Anual }\end{array}$ & Total \\
\hline $\begin{array}{l}\text { Corporación Universitaria Minuto } \\
\text { de Dios }\end{array}$ & Bogotá & $\begin{array}{l}\text { Maestría en } \\
\text { educación }\end{array}$ & Privado & 5 semestres & $3,507,000$ & $7,014,000$ & $17,535,00$ \\
\hline Pontificia Universidad Javeriana & Bogotá & $\begin{array}{l}\text { Maestría en } \\
\text { educación }\end{array}$ & Privado & 4 semestres & $6,965,000$ & $13,9330,00$ & $27,860,000$ \\
\hline Universidad Antonio Nariño & Bogotá & $\begin{array}{l}\text { Maestría en } \\
\text { educación }\end{array}$ & Privado & 4 semestres & $6,933,000$ & $13,866,000$ & $27,732,000$ \\
\hline $\begin{array}{l}\text { Universidad Cooperativa de } \\
\text { Colombia }\end{array}$ & Bogotá & $\begin{array}{l}\text { Maestría en } \\
\text { educación }\end{array}$ & Privado & 4 semestres & $3,815,300$ & $7,630.600$ & $15,261,000$ \\
\hline Universidad de Antioquia & Medellín & $\begin{array}{l}\text { Maestría en } \\
\text { educación }\end{array}$ & Público & 4 semestres & $5,515,640$ & $11,031,280$ & $22,062,560$ \\
\hline Universidad de Caldas & Manizales & $\begin{array}{l}\text { Maestría en } \\
\text { educación }\end{array}$ & Público & 4 semestres & $3,972,058$ & $7,584,115$ & $15,168,230$ \\
\hline Universidad de la Sabana & Bogotá & $\begin{array}{l}\text { Maestría en } \\
\text { educación }\end{array}$ & Privado & 4 semestres & $7,700,000$ & $15,400,00$ & $30,800,000$ \\
\hline Universidad de los Andes & Bogotá & $\begin{array}{l}\text { Maestría en } \\
\text { educación }\end{array}$ & Privado & 4 semestres & $8,550,000$ & $17,100,000$ & $34,200,000$ \\
\hline Universidad de Manizales & Manizalez & $\begin{array}{l}\text { Maestría en } \\
\text { educación }\end{array}$ & Privado & 4 semestres & $3,801,000$ & $7,602,000$ & $15,204,000$ \\
\hline Universidad de san Buenaventura & Bogotá & $\begin{array}{l}\text { Maestría en } \\
\text { ciencias }\end{array}$ & Privado & 4 semestres & $5,277,000$ & $10,554,000$ & $21,108,000$ \\
\hline Universidad del Norte & Barranquilla & $\begin{array}{l}\text { Maestría en } \\
\text { educación }\end{array}$ & Privado & 4 semestres & $6,077,320$ & $12,154,640$ & $24,309,280$ \\
\hline
\end{tabular}




\begin{tabular}{|c|c|c|c|c|c|c|c|}
\hline Institución & Ciudad & Programa & Carácter & Duración & $\begin{array}{c}\text { Precio } \\
\text { Semestral }\end{array}$ & $\begin{array}{l}\text { Precio } \\
\text { Anual }\end{array}$ & Total \\
\hline Universidad El Bosque & Bogotá & $\begin{array}{l}\text { Maestría en } \\
\text { docencia }\end{array}$ & Privado & 4 semestres & $5,936,000$ & $11,872,000$ & $23,744,000$ \\
\hline $\begin{array}{l}\text { Universidad Externado de } \\
\text { Colombia }\end{array}$ & Bogotá & $\begin{array}{l}\text { Maestría en } \\
\text { educación }\end{array}$ & Privado & 4 semestres & $4,200,000$ & $8,400,000$ & $16,800,000$ \\
\hline $\begin{array}{l}\text { Universidad Francisco José de } \\
\text { Caldas }\end{array}$ & Bogotá & $\begin{array}{l}\text { Maestría en } \\
\text { educación }\end{array}$ & Público & 4 semestres & $3,447,270$ & $6,894,540$ & $13,789,080$ \\
\hline Universidad ICESI & Cali & $\begin{array}{l}\text { Maestría en } \\
\text { educación }\end{array}$ & Privado & 4 semestres & $6,310,000$ & $12,620,000$ & $25,240,000$ \\
\hline $\begin{array}{l}\text { Universidad Nacional de } \\
\text { Colombia }\end{array}$ & Bogotá & $\begin{array}{l}\text { Maestría en } \\
\text { educación }\end{array}$ & Público & 4 semestres & $4,099,120$ & $8,198,240$ & $16,396,480$ \\
\hline Universidad Pedagógica Nacional & Bogotá & $\begin{array}{l}\text { Maestría en } \\
\text { educación }\end{array}$ & Público & 4 semestres & $3,447,275$ & $6,894,550$ & $13,789,100$ \\
\hline $\begin{array}{l}\text { Universidad Pedagógica y } \\
\text { Tecnológica de Colombia }\end{array}$ & Tunja & $\begin{array}{l}\text { Maestría en } \\
\text { educación }\end{array}$ & Público & 4 semestres & $4,136,730$ & $8,273,460$ & $16,546,920$ \\
\hline Universidad Pontificia Bolivariana & Medellín & $\begin{array}{l}\text { Maestría en } \\
\text { educación }\end{array}$ & Privado & 4 semestres & $5,837,000$ & $11,674,000$ & $23,348,000$ \\
\hline $\begin{array}{l}\text { Universidad Tecnológica de } \\
\text { Pereira }\end{array}$ & Bogotá & $\begin{array}{l}\text { Maestría en } \\
\text { educación }\end{array}$ & Público & 4 semestres & $4,481,457$ & $8,962,914$ & $17,925,828$ \\
\hline Promedios & & & & & $5,191,408$ & $10,382,817$ & $20,940,984$ \\
\hline
\end{tabular}

Anexo 2, Tabla 4. Diferenciación público-privado

\begin{tabular}{|c|c|c|c|c|}
\hline \multirow{2}{*}{ Institución } & \multicolumn{2}{|c|}{ Semestral } & \multicolumn{2}{|c|}{ Total } \\
\hline & Privado & Público & Privado & Público \\
\hline Corporación Universitaria Minuto de Dios & $\$ 3,507,000$ & & $\$ 14,028,000$ & \\
\hline Pontificia Universidad Javeriana & $\$ 6,965,000$ & & $\$ 27,860,000$ & \\
\hline Universidad Antonio Nariño & $\$ 6,933,000$ & & $\$ 27,732,000$ & \\
\hline Universidad Cooperativa de Colombia & $\$ 3,815,300$ & & $\$ 15,261,200$ & \\
\hline Universidad de Antioquia & & $\$ 5,515,640$ & & $\$ 22,062,560$ \\
\hline Universidad de Caldas & & $\$ 3,792,058$ & & $\$ 15,168,230$ \\
\hline Universidad de la Sabana & $\$ 7,700,000$ & & $\$ 30,800,000$ & \\
\hline Universidad de los Andes & $\$ 8,550,000$ & & $\$ 34,200,000$ & \\
\hline Universidad de Manizales & $\$ 3,801,000$ & & $\$ 15,204,000$ & \\
\hline Universidad de san Buenaventura & $\$ 5,277,000$ & & $\$ 21,108,000$ & \\
\hline Universidad del Norte & $\$ 6,077,320$ & & $\$ 24,309,280$ & \\
\hline Universidad El Bosque & $\$ 5,936,000$ & & $\$ 23,744,000$ & \\
\hline Universidad Externado de Colombia & $\$ 4,200,000$ & & $\$ 16,800,000$ & \\
\hline Universidad Francisco José de Caldas & & $\$ 3,447,270$ & & $\$ 13,789,080$ \\
\hline Universidad ICESI & $\$ 6,310,000$ & & $\$ 25,240,000$ & \\
\hline Universidad Nacional de Colombia & & $\$ 4,099,120$ & & $\$ 16,396,480$ \\
\hline Universidad Pedagógica Nacional & & $\$ 3,447,275$ & & $\$ 13,789,100$ \\
\hline Universidad Pedagógica y Tecnológica de & & & & \\
\hline Colombia & & $\$ 4,136,730$ & & $\$ 16,546,920$ \\
\hline Universidad Pontificia Bolivariana & $\$ 5,837,000$ & & $\$ 23,348,000$ & \\
\hline Universidad Tecnológica de Pereira & & $\$ 4,481,487$ & & $\$ 17,925,948$ \\
\hline Promedios & $\$ 5,762,202$ & $\$ 4,131,369$ & $\$ 23,048,806$ & $\$ 16,525,474$ \\
\hline
\end{tabular}


Anexo 3, Tabla 5. PIB per cápita Colombia, Chile y Perú

\begin{tabular}{lcrrl}
\hline & PIB 2015 Precios corrientes & Población & PIB per cápita & \multicolumn{1}{c}{ Magnitud } \\
\hline Colombia (PIB miles de millones de pesos) & 800,849 & $47,791,393$ & $16,757,181$ & Peso colombiano \\
\hline Perú (PIB millones de nuevos soles) & 606,406 & $30,814,175$ & 19,679 & Nuevos soles \\
\hline Chile (PIB millones de pesos) & $118,535,235$ & $17,762,647$ & $6,673,287$ & Pesos chileno \\
\hline
\end{tabular}

Anexo 4, Tabla 6. Maestrías chilenas y peruanas

\begin{tabular}{|c|c|c|c|c|c|c|}
\hline \multirow{2}{*}{ Institución } & \multirow{2}{*}{ Programa } & \multirow{2}{*}{ País } & \multirow{2}{*}{ Carácter } & \multicolumn{2}{|c|}{ Precio } & \multirow{2}{*}{$\begin{array}{c}\text { Promedio } \\
\text { Anual }\end{array}$} \\
\hline & & & & Anula & Total & \\
\hline Universidad de Chile & Magister en educación & Chile & Público & $\$ 3,147,900$ & $\$ 6,295,800$ & $\$ 2,320,967$ \\
\hline Universidad de Santiago de Chile & Magister en educación & Chile & Público & $\$ 2,000,000$ & $\$ 4,000,000$ & \\
\hline Universidad de la Frontera & Magister en educación & Chile & Público & $\$ 1,815,000$ & $\$ 3,630,000$ & \\
\hline Pontificia Universidad Católica de Chile & Magister en educación & Chile & Privado & $\$ 2,400,000$ & $\$ 4,800,000$ & $\$ 1,691,500$ \\
\hline Pontificia Universidad Católica de Valparaiso & Magister en educación & Chile & Privado & $\$ 1,500,000$ & $\$ 3,000,000$ & \\
\hline Universidad de Concepción & Magister en educación & Chile & Privado & $\$ 1,174,500$ & $\$ 2,349,000$ & \\
\hline Pontificia Universidad Católica del Perú & Maestría en educación & Perú & Privado & $\mathrm{S} / .11,730$ & $\mathrm{~S} / .23,460$ & $\mathrm{~S} / .8,029$ \\
\hline Universidad Peruana Cayetano Heredia & Maestría en educación & Perú & Privado & $\mathrm{S} / .9,958$ & $\mathrm{~S} / .19,916$ & \\
\hline Universidad del Señor de Sipan & Maestría en educación & Perú & Privado & $S / .2,400$ & S/.4,800 & \\
\hline Universidad Nacional Mayor de San Marcos & Maestría en educación & Perú & Público & $\mathrm{S} / .8,096$ & S/.16,192 & S/.5,419 \\
\hline Universidad Nacional San Antonio Abad del Cusco & Maestría en educación & Perú & Público & S/.4,800 & S/.4,800 & \\
\hline Universidad Nacional de Trujillo & Maestría en educación & Perú & Público & $\mathrm{S} / .3,360$ & S/.6,720 & \\
\hline
\end{tabular}

Anexo 5, Tabla 7. Promedio costos anuales

\begin{tabular}{ccc}
\hline \multirow{2}{*}{ Pais } & \multicolumn{2}{c}{ Carácter } \\
\cline { 2 - 3 } & Privado & Público \\
\hline Colombia & $\$ 11,524,403$ & $\$ 8,262,737$ \\
\hline Chile & $\$ 1,691,500$ & $\$ 2,320,927$ \\
\hline Perú & $\mathrm{S} / .8,029$ & $\mathrm{~S} / .5,419$ \\
\hline
\end{tabular}

Anexo 6, Tabla 8. Porcentaje del ingreso per cápita

\begin{tabular}{ccc}
\hline \multirow{2}{*}{ Pais } & \multicolumn{2}{c}{ Carácter } \\
\cline { 2 - 3 } & Privado & Público \\
\hline Colombia & $68.77 \%$ & $49.31 \%$ \\
\hline Chile & $25.35 \%$ & $34.78 \%$ \\
\hline Perú & $40.80 \%$ & $27.54 \%$ \\
\hline
\end{tabular}

Anexo 7, Gráfico 7. Maestrías en Colombia

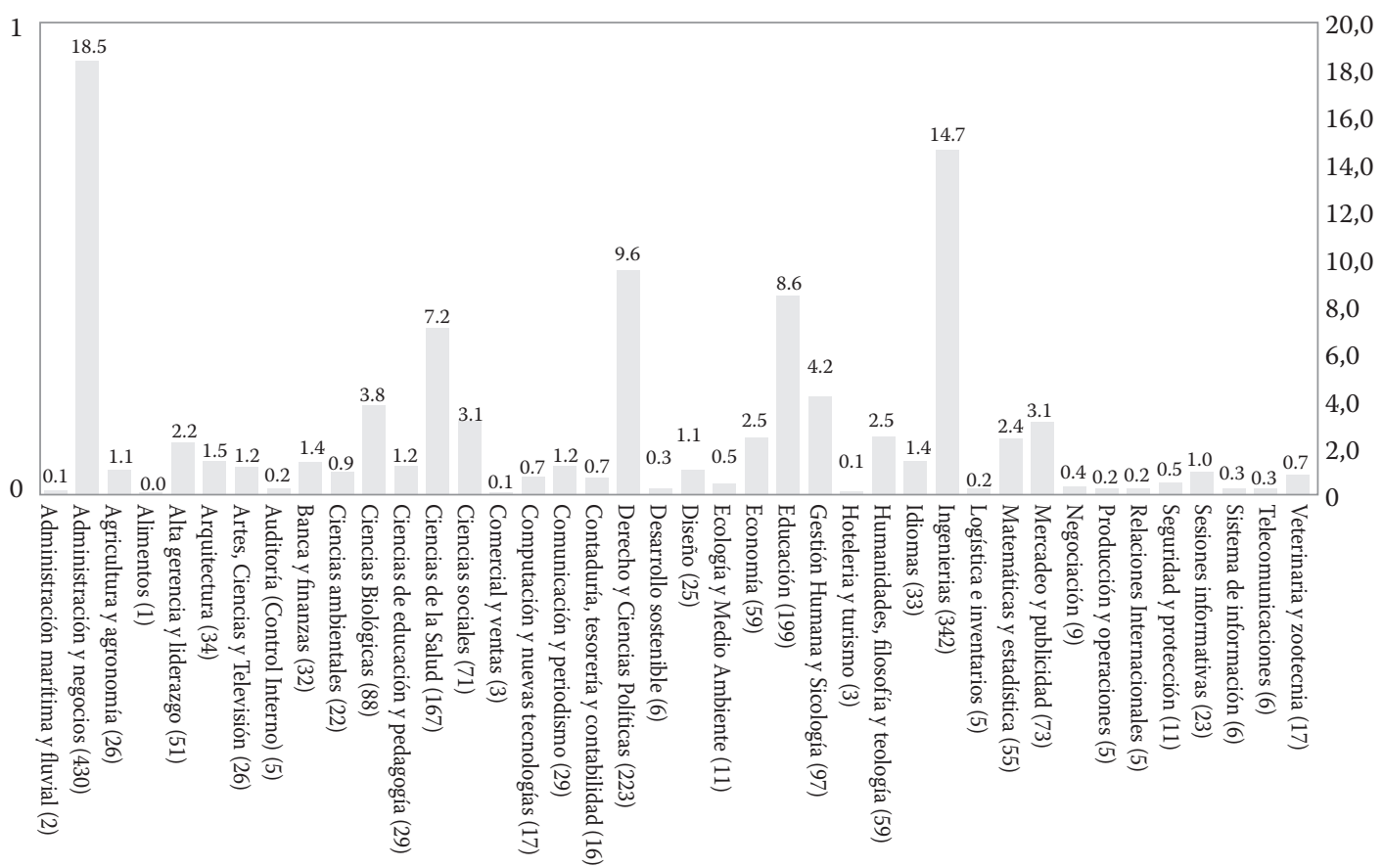

Universidad Antonio Nariño • Facultad de Ciencias de la Educación 